\title{
Two-parameter Alteration of B-splines
}

\author{
Yajuan Li \\ School of Science \\ Hangzhou Dianzi University \\ Hangzhou, China,310018
}

\author{
Mingzeng Yang \\ Mathmatics department \\ Henan Institute of Education \\ Zhengzhou, China, 450014
}

\begin{abstract}
Altering one or more knots of B-spline curves and surfaces, points of curves and surfaces will move on well defined curves called paths. Letting the knot value tend to infinity, the limit of the paths will be computed in this paper. The effect of two-parameter alteration of B-splines is discussed.
\end{abstract}

Keywords-B-spline surface, NURBS surface, knot modification, extended paths

\section{INTRODUCTION}

A B-spline curve is the composition of a number of curve segments, each of which is defined on a knot span[1]. As is well known, B-spline curves and surfaces(and their rational forms-NURBS curves and NURBS surfaces) are widely used in Computer Aided Geometric Design and Computer Graphics(CAGD\&CG). In the designing process, one of the most important thing is modification of the shape of the model. Due to the popularity of the B-spline and NURBS models, there are many methods to modify the shape of the B-splines[2-12], among which modifying the knots is an important way.

Modifying knots of B-splines, the association between curve segments and knot spans will be changed, and hence the shape of the curve will be modified. For example, changing one or more knots of a B-spline curve, points of the curve will move on well defined curves called paths[6]. There are some publications studied the effect of the alteration of one or more knots on the shape of the curve[610]. By these theories, some authors worked on the constraint-based shape modification of B-spline curves and NURBS curves [11-13]. [14-16] performed the research on the effect of the alteration of some knots of B-spline curves and surfaces and obtained some interesting results on the restricted part of the path.

In this paper, we present some theorems describing the effect of alteration of knots of B-spline and NURBS surfaces based on the modification of two parameters.

This paper is organized as follows: In Section 2, the basic definitions and notations will be presented. Then in Section 3, we present the effect of the modification of two knots of NURBS curves by one parameter. Alteration of knots of tensor surfaces by two parameter will be given in Section 4 and 5.

\section{Modify The KNOTS OF B-Splines}

\section{A. B-SPLINES}

A B-spline is a generalization of the Bézier curve. Let a vector known as the knot vector be defined $U=\{\mathrm{ul}\}^{+\infty}$, where $U$ is a nondecreasing sequence. The basic definitions of basis functions are the followings.

Definition 1. Let $U=\{\mathrm{ul}\}^{+\infty}$ be a given knot sequence with $\mathrm{ul} \leq \mathrm{ul}+1$, the normalized B-spline basis functions of order $\mathrm{k}$ (degree $\mathrm{k}-1$ ) are defined recursively by the following equations:

$$
\begin{aligned}
& N_{l, 1}=\left\{\begin{array}{l}
1, \text { if } u \in\left[u_{l}, u_{l+1}\right) \\
0, \text { if otherwise. }
\end{array}\right. \\
& N_{l, k}(u)=\frac{u-u_{l}}{u_{l+k-1}-u_{l}} N_{l, k-1}(u)+\frac{u_{l+k}-u}{u_{l+k}-u_{l+1}} N_{l+1, k-1}(u)
\end{aligned}
$$

Here we define $0 / 0=0$. Define control points $\mathrm{pl}, 1=0$, $1, \cdots, \mathrm{n}$, then the curve defined by

$$
\boldsymbol{r}(u)=\sum_{l=0}^{n} N_{l . k}(u) \boldsymbol{p}_{l}, \quad u \in\left[u_{k-1}, u_{n+1}\right]
$$

is a B-spline curve. The ith arc can be written as

$$
\boldsymbol{r}_{i}(u)=\sum_{l=i-k+1}^{i} N_{l . k}(u) \boldsymbol{p}_{l}, u \in\left[u_{i}, u_{i+1}\right],{ }_{k-1 \leq i \leq n} .
$$

Defining weights wl, we get the definition of Non Uniform Rational B-spline(NURBS) curve

$$
\boldsymbol{r}(u)=\frac{\sum_{l=0}^{n} N_{l, k}(u) w_{l} \boldsymbol{p}_{l}}{\sum_{l=0}^{n} N_{l, k}(u) w_{l}}, u \in\left[u_{k-1}, u_{n+1}\right]
$$

The definition of B-spline curve and NURBS curve can be generalized to the tensor product surfaces:

Definition 2. The surface $s(u, v)$ defined by

$$
\begin{gathered}
\boldsymbol{s}(u, v)=\sum_{l=0}^{n} \sum_{g=0}^{m} N_{l, k}(u) N_{g, h}(v) \boldsymbol{p}_{l g}, \\
u \in\left[u_{k-1}, u_{n+1}\right], v \in\left[v_{h-1}, v_{m+1}\right]
\end{gathered}
$$

is called B-spline surface of order $\mathrm{k} \times \mathrm{h}$, where N1,k(u) and $\mathrm{Ng}, \mathrm{h}(\mathrm{v})$ are B-spline basis functions of order $\mathrm{k}$ and $\mathrm{h}$, which defined on given knot sequences $U=\{\mathrm{ul}\}^{+\infty}$ and $\mathrm{V}$ $=\{\mathrm{vg}\}^{+\infty}$ respectively, and plg are control points. The $\mathrm{i} \times$ $\mathrm{j}$ 'th patch can be written as 


$$
\boldsymbol{s}_{i j}(u, v)=\sum_{l=i-k+1}^{i} \sum_{g=j-h+1}^{j} N_{l, k}(u) N_{g, h}(v) \boldsymbol{p}_{l g},
$$

Define weights wlg, we have the definition of the NURBS surface

$$
\boldsymbol{s}(u, v)=\frac{\sum_{l=0}^{n} \sum_{g=0}^{m} N_{l, k}(u) N_{g, h}(v) w_{l g} \boldsymbol{p}_{l g}}{\sum_{l=0}^{n} \sum_{g=0}^{m} N_{l, k}(u) N_{g, h}(v) w_{l g}}
$$

\section{B. one-parameter alteration of $b \_$spline curves}

The knot vector is a nondecreasing sequence, so when a knot ui is altered within the defined range [ui-1, ui+1], points of the B-spline curve will move on special rational curves $\mathrm{s}(\mathrm{u}$, ui) called paths[6]. When we modify two knots ui and uj, $\mathrm{i}<$ $\mathrm{j}$ to the values ui $+\mathrm{t} \lambda$ and $\mathrm{uj}-\mathrm{t} \lambda$ by one parameter $\lambda$ with $\mathrm{t}$ $\in(0 ; 1)$, the value of $\lambda$ must be within the range $[-\mathrm{c} / \mathrm{t} ; \mathrm{c} / \mathrm{t}], \mathrm{c}$ $=\min \{$ ui - ui-1, ui $+1-$ ui, uj - uj- 1 , uj $+1-$ uj $\}$. So paths obtained by the modification of knots are relatively short arcs due to the limited range of the modification. To get more information about spline's characteristics, some authors extended the paths by extending the value of the modified knot[16], that is, modifying ui in any way allowing ui $<$ ui- 1 and ui $>$ ui +1 and $\lambda$ can be any value. In [15], the authors presented a limit theorem of one-parameter alteration of two knots of a B-spline curve:

Lemma 1 Modifying the knots $u i=u i+t \lambda, u i+z=u i+z-$ (1-t) $\lambda, z=1,2, \cdots, k$ of a B-spline curve, the points of the extended paths of the $\operatorname{arcs} r(u), u \in[u i ; u i+z)$ tend to a point of the line segment pi pi+z-k, the barycentric coordinates of which are $t$ and $1-t$, i.e.

$$
\lim _{\lambda \rightarrow-\infty} \boldsymbol{r}(u, t, \lambda)=t \boldsymbol{p}_{i}+(1-t) \boldsymbol{p}_{i+z-k}, u \in\left[u_{i}, u_{i+z}\right), t \in[0,1] .
$$

\section{One-Parameter Alteration Of NURBS Curves}

By a parameter $\mathrm{t} \in[0,1]$, we can modify two knots of a NURBS curve in the same way. The extended paths is obtained:

$$
\boldsymbol{r}(u, t, \lambda)=\frac{\sum_{l=0}^{n} N_{l, k}(u, t, \lambda) w_{l} \boldsymbol{p}_{l}}{\sum_{l=0}^{n} N_{l, k}(u, t, \lambda) w_{l}}, u \in\left[u_{k-1}, u_{n+1}\right] .
$$
have

Denoting wl pl by ql and letting $\lambda \rightarrow-\infty$, by Lemma 1 , we

$$
\begin{aligned}
& \lim _{\lambda \rightarrow-\infty} \sum_{l=0}^{n} N_{l, k}(u, t, \lambda) w_{l}=t w_{i}+(1-t) w_{i+z-k} \\
& \lim _{\lambda \rightarrow-\infty} \sum_{l=0}^{n} N_{l, k}(u, t, \lambda) \boldsymbol{q}_{l}=t \boldsymbol{q}_{i}+(1-t) \boldsymbol{q}_{i+z-k}=t w_{i} \boldsymbol{p}_{i}+(1-t) w_{i+z-k} \boldsymbol{p}_{i+z-k}
\end{aligned}
$$

Thus we have the following proposition:

Theorem 1 Modifying the knots ui $=u i+t \lambda$, $u i+z=u i+z$ -(1-t) $\lambda, z=1,2, \cdots, k$ of a NURBS curve, the points of the extended paths of the $\operatorname{arcs} r(u), u \in[u i, u i+z)$ tend to a point of the line segment pi pi+z-k, the barycentric coordinates of which are twi and $(1-t) w i+z-k$, i.e.(See Fig. 1)

$$
\lim _{\lambda \rightarrow-\infty} \boldsymbol{r}(u, t, \lambda)=t w_{i} \boldsymbol{p}_{i}+(\mathbf{1}-\mathbf{t}) w_{i+z-k} \boldsymbol{p}_{i+z-k}, u \in\left[u_{i}, u_{i+z}\right), t \in[0,1] .
$$
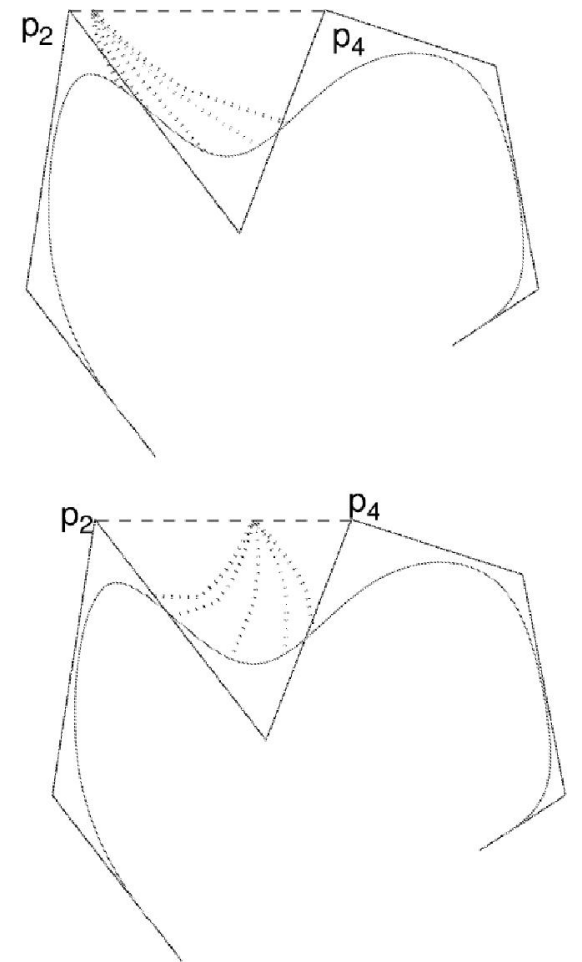

Figure 1. Extended paths of the points of a cubic NURBS curve obtained by a one-parameter

modification of $\mathrm{u} 4$ and $\mathrm{u} 6$ with $\mathrm{t}=0.2$ (left) and $\mathrm{t}=0.6$ (right).

\section{Two-Parameter Alteration Of B-Spline SURFACES}

Modifying four knots of a B-spline surface by two parameter $\mathrm{t}$ and $\mathrm{f}, 0 \leq \mathrm{t}, \mathrm{f} \leq 1$, that is, changing the values of ui, $\mathrm{ui}+\mathrm{a},(\mathrm{a}=1,2, \ldots, \mathrm{k})$ and $\mathrm{vj}, \mathrm{vj}+\mathrm{b},(\mathrm{b}=1,2, \ldots, \mathrm{h})$ to $\mathrm{u},+\mathrm{t} \lambda, \mathrm{ui}+\mathrm{a}-$ (1-t) $\lambda$ and $v j+f \mu, v j+b-(1-f) \mu$ respectively, the extended paths of patches $s(u, v, t, f, \lambda, \mu), u \in[u i, u i+a], v \in[v j, v j+b]$ can be expressed as:

$$
\boldsymbol{s}(u, v, t, f, \lambda, \mu)=\sum_{l=i-k+1}^{i+a-1} \sum_{g=j-h+1}^{j+b-1} N_{l, k}(u, t, \lambda) N_{g, h}(v, f, \mu) \boldsymbol{p}_{l g}
$$

Here we give the following proposition:

Theorem 2. Altering four knots ui, ui+a, $(\mathrm{a}=1,2, \cdots, \mathrm{k})$ and $v j, v j+b,(b=1,2, \cdots, h)$ of a B-spline surface by two parameter $\mathrm{t}, \mathrm{f}$, the extended paths of points of patches are

$$
\lim _{\substack{\lambda \rightarrow-\infty \\ \mu \rightarrow-\infty}} \boldsymbol{s}(u, v, t, f, \lambda, \mu)=t\left(f \boldsymbol{p}_{i j}+(1-f) \boldsymbol{p}_{i, j+b-h}\right)+(1-t)\left(f \boldsymbol{p}_{i+a-k, j}+(1-f) \boldsymbol{p}_{i+a-k, j+b-h}\right)
$$

Proof: From Section 2 of [15], we know that the following equations hold for $\mathrm{u} \in[\mathrm{ui}, \mathrm{ui}+\mathrm{a})$ :

$$
\begin{aligned}
& \lim _{\lambda \rightarrow-\infty} N_{i, k}(u, t, \lambda)=t, \lim _{\lambda \rightarrow-\infty} N_{i+a-k, k}(u, t, \lambda)=1-t, a=1, \ldots, k-1, \\
& \lim _{\lambda \rightarrow-\infty} N_{i, k}(u, t, \lambda)=\lim _{\lambda \rightarrow-\infty} N_{i+a-k, k}(u, t, \lambda)=1, a=k .
\end{aligned}
$$

It is the same for the v-direction:

$$
\lim _{\mu \rightarrow-\infty} N_{j, h}(v, f, \mu)=f, \lim _{\lambda \rightarrow-\infty} N_{j+b-h, h}(v, f, \mu)=1-f, b=1, \ldots, h-1 \text {, }
$$


$\lim _{\mu \rightarrow-\infty} N_{j, h}(v, f, \mu)=\lim _{\lambda \rightarrow-\infty} N_{j+b-h, h}(v, f, \mu)=1, b=h$

The above equations yield (2) (See Fig. 2).

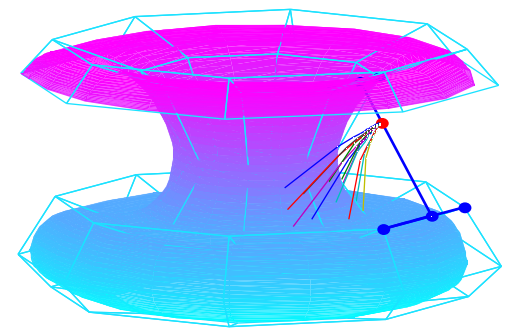

Figure 2. Extended paths of the points of a $3 \times 3$ B-spline surface obtained

by two parameter alteration $(\mathrm{t}=0.3, \mathrm{f}=0.6)$

\section{Two-Parameter Alteration Of NURBS SURFACES}

Substituting wlgplg by qlg in (1) and modifying four knots of a NURBS surface by two parameter $t$ and $f, 0 \leq t, f \leq$ 1 as in section 4 , we get the extended paths of the segments $\mathrm{s}(\mathrm{u}, \mathrm{v}, \mathrm{t}, \mathrm{f}, \lambda, \mu),(\mathrm{u} \in[\mathrm{ui}, \mathrm{ui}+\mathrm{a}], \mathrm{v} \in[\mathrm{vj}, \mathrm{vj}+\mathrm{b}])$ of the NURBS surface

$$
\boldsymbol{s}(u, v, t, f, \lambda, \mu)=\frac{\sum_{l=i-k+1}^{i+a-1} \sum_{g=j-h+1}^{j+b-1} N_{l, k}(u, t, \lambda) N_{g, h}(v, f, \mu) \boldsymbol{q}_{l g}}{\sum_{l=i-k+1}^{i+a-1} \sum_{g=j-h+1}^{j+b-1} N_{l, k}(u, t, \lambda) N_{g, h}(v, f, \mu) w_{l g}}
$$

By Theorem 2, the numerator and the denominator of the limitation of $\mathrm{s}(\mathrm{u}, \mathrm{v}, \mathrm{t}, \mathrm{f}, \lambda, \mu)$ when $\lambda \rightarrow-\infty$ and $\mu \rightarrow-\infty$ are obtained below:

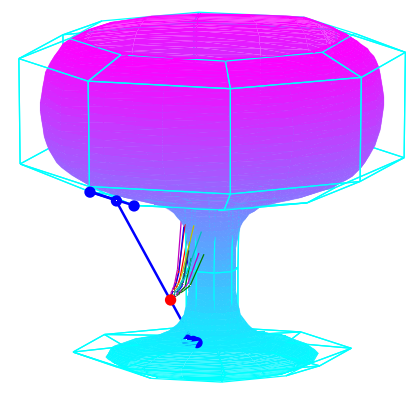

Figure 3. Extended paths of the points of a $3 \times 3$ NURBS surface obtained

$$
\text { by two parameter alteration }(\mathrm{t}=0.7, \mathrm{f}=0.7)
$$

$$
\begin{aligned}
& \lim _{\substack{\lambda \rightarrow-\infty \\
\mu \rightarrow-\infty}} \sum_{l=i-k+1}^{i+a-1} \sum_{g=j-h+1}^{j+b-1} N_{l, k}(u, t, \lambda) N_{g, h}(v, f, \mu) \boldsymbol{q}_{l g} \\
& =t\left(f \boldsymbol{q}_{i j}+(1-f) \boldsymbol{q}_{i, j+b-h}\right)+(1-t)\left(f \boldsymbol{q}_{i+a-k, j}+(1-f) \boldsymbol{q}_{i+a-k, j+b-h}\right) \\
& \lim _{\substack{\lambda \rightarrow-\infty \\
\mu \rightarrow-\infty}} \sum_{l=i-k+1}^{i+a-1} \sum_{g=j-h+1}^{j+b-1} N_{l, k}(u, t, \lambda) N_{g, h}(v, f, \mu) w_{l g} \\
& =t\left(f w_{i j}+(1-f) w_{i, j+b-h}\right)+(1-t)\left(f w_{i+a-k, j}+(1-f) w_{i+a-k, j+b-h}\right)
\end{aligned}
$$

Therefore, we get the theorem for the two-parameter alteration of four knots of NURBS surfaces:

Theorem 3. Altering four knots ui, ui $+\mathrm{a},(\mathrm{a}=1,2, \cdots, \mathrm{k})$ and $v j, v j+b,(b=1,2, \cdots, h)$ of a NURBS surface by two parameter $\mathrm{t}, \mathrm{f}, 0 \leq \mathrm{t}, \mathrm{f} \leq 1$, the extended paths of points of patches $s(u, v, t, f, \lambda, \mu)$ converge to a point when $\lambda \rightarrow-\infty$ and $\mu \rightarrow-\infty$, that is:

$$
\begin{aligned}
& \lim _{\substack{\lambda \rightarrow-\infty \\
\mu \rightarrow-\infty}} s(u, v, t, f, \lambda, \mu) \\
& =\frac{t\left(f \boldsymbol{q}_{i j}+(1-f) \boldsymbol{q}_{i, j+b-h}\right)+(1-t)\left(f \boldsymbol{q}_{i+a-k, j}+(1-f) \boldsymbol{q}_{i+a-k, j+b-h}\right)}{t\left(f w_{i j}+(1-f) w_{i, j+b-h}\right)+(1-t)\left(f w_{i+a-k, j}+(1-f) w_{i+a-k, j+b-h}\right)} \\
& \quad \text { where qlg }=\text { wlgplg, } 1=\mathrm{i}, \mathrm{i}+\mathrm{a}-\mathrm{k}, \mathrm{g}=\mathrm{j}, \mathrm{j}+\mathrm{b}-\mathrm{h} .
\end{aligned}
$$

\section{ACKNOWLEDGMENT}

This work was Supported by the National Natural Science Foundation of China (No.60970079) and National Natural Science Foundation of China, Tian Yuan Special Foundation (No.11026107).

\section{REFERENCES}

[1] GORDON, W.J.;;RIESENFELD, R.F. B-spline curves and surfaces, In: Barnhill,R.E. and Riesenfeld, R.F. eds., Computer Aided Geometric Design[M]. Academic Press, New York, 1974, PP:95-126.

[2] AU C.K.; YUEN M.M.F. Unified Approach to NURBS Curve Shape Modification. Computer Aided Design [J], 1995, 27(2), PP: 85-93.

[3] FOWLER Barry M.; BARTELA, Richard H. Constraint-based curve manipulation. IEEE Computer Graphics and Applications [J], 1993, 13(5), PP:43-49.

[4] JAVIER Sanchez-Reyes. A Simple Technique for NURBS Shape Modification. IEEE Computer Graphics and Applications [J], 1997,17(1), PP: 52-59.

[5] L. Piegl. Modifying the Shape of Rational B-Splines. Part 1: Curves, Computer Aided Design [J], 1989, 21(8), PP: 509-518.

[6] JUHASZ Imre, HOFFMANN Miklos. The effect of knot modifications on the shape of B-spline curves. Journal for Geometry and Graphics [J], 2001, 5, PP: 111-119.

[7] JUHASZ Imre, HOFFMANN Miklos Miklos. Modifying a knot of B-spline curves. Computer Aided Geometric Design [J], 2003, 20, PP: 243-245.

[8] HOFFMANN Miklos, JUHASZ Imre. On the knot modification of a B-spline curve. Publicationes Mathematicae Debrecen [J], 2004, 65, PP: 193-203.

[9] LI Ya-juan; WANG Guo-zhao. On knot modifications of B-spline or NURBS surface, Journal of Computer-Aided Design and Computer Graphics, 2005, 17(5) , PP: 986-989.

[10] JUHASZ Imre, HOFFMANN Miklos. On the quartic curve of Han. Journal of Computational and Applied Mathematics [J], 2009, 223(1), PP: 124-132.

[11] JUHASZ Imre, HOFFMANN Miklos. On the family of B-spline surfaces obtained by knot modification. Mathematical communications [J], 2006, 11, PP: 9-16.

[12] JUHASZ Imre. A shape modification of B-spline curves by symmetric translation of two knots. Acta. Acad. Paed. Agriensis, Sect [J], 2001, 28, PP: 69-77.

[13] JUHASZ Imre, HOFFMANN Miklos. Constrained shape modification of cubic B-spline curves by means of knots. ComputerAided Design [J], 2004, 36, 437-445.

[14] LI Ya-juan, WANG Guo-zhao. Constrained shape modification of cubic NURBS curve based on knots and weights. Applied Mathematics-A Journal of Chinese Universities [J], 2006, 21(1), PP: 118-124. 
[15] HOFFMANN Miklos, JUHASZ Imre. A limit theorem for oneparameter alteration of two knots of B-spline curves. Annales Mathematicae et Informaticae[J], 2006, 32, PP: 56-60.

[16] HOFFMANN Miklos, JUHASZ Imre. Symmetric alteration of two knots of B-spline curves. Journal for Geometry and Graphics[J], 2005, 9(1), 43-49.
[17] LI Ya-juan; WANG Guo-zhao. Symmetric alteration of four knots of Bspline and NURBS surfaces. Journal of Zhejiang University SCIENCE A[J], 2006, 7(6), PP: 1084-1087.

[18] Ulker E., Arslan A.. Automatic knot adjustment using an artificial immune system for B-spline curve approximation. Information Sciences[J], 2009, 179(10) Sp., PP: 1483-1494. 